\title{
Spectrum of clinical signs and genetic characterization of Gelatinous Drop- like Corneal Dystrophy in a Colombian Family
}

${ }^{1}$ Sara Morantes, ${ }^{3}$ Cerys J. Evans,,${ }^{6,7}$ Ana V. Valencia, ${ }^{3}$ Alice E. Davidson, ${ }^{3}$ Alison J. Hardcastle, ${ }^{4}$ Andrés Ruiz Linares, ${ }^{3,5}$ Stephen J. Tuft, ${ }^{1},{ }^{2}$ Miguel Cuevas

${ }^{1}$ Ophthalmology Section, Faculty of Medicine Universidad de Antioquia, Carrera 51d № 62-29, Medellín, Colombia.

${ }^{2}$ Cornea and Ocular Surface Service. Hospital Universitario San Vicente Fundación. Medellín, Colombia.

${ }^{3}$ UCL Institute of Ophthalmology, 11-43 Bath Street, London, EC1V 9EL, UK

${ }^{4}$ Department of Genetics, Evolution and Environment, University College London, Gower Street, London WC1E 6BT, UK

${ }^{5}$ Moorfields Eye Hospital, 162 City Road, London EC1V 2PD, UK ${ }^{6}$ Instituto de Investigaciones Médicas. Facultad de Medicina, Universidad de Antioquia, carrera 51d № 62-29, Medellín, Colombia

${ }^{7}$ Facultad de Medicina, Universidad Pontificia Bolivariana, calle 78b № $72^{\mathrm{a}}-109$, Medellín, Colombia.

Corresponding author:

Miguel Cuevas, MD. Calle 64 NN$^{\circ} 51$ D - 154. Hospital Universitario San Vicente Fundación. BI 8. Medellín, Colombia. dr.cuevasoftalmo@gmail.com 


\begin{abstract}
Purpose: To describe the clinical signs of gelatinous drop-like corneal dystrophy (GDCD) in a consanguineous Colombian family and determine the underlying genetic cause.
\end{abstract}

Methods: We performed ocular examination of available family members and bidirectionally Sanger sequenced the GDCD-associated gene, TACSTD2. In one individual, the presence of subepithelial amyloid was confirmed with biopsy.

Results: The parents were consanguineous and five of their ten children had GDCD. Typical mulberry subepithelial deposits with subepithelial vascularization were present in 3 individuals; 2 individuals only had mild polymorphic anterior stromal opacity. We identified a homozygous TACSTD2 missense mutation, c.551A>G, p.(Tyr184Cys), in affected family members. Both parents were heterozygous for the mutation, and unaffected siblings were either heterozygous or homozygous wild-type for this allele. In the Colombian population this mutation has a minor allele frequency (MAF) of $0.53 \%$.

Conclusion: The clinical presentation of GDCD in this family was variable and does not solely support an age dependent progression of the phenotype, suggesting that environmental or other genetic factors can modify phenotypic expression. The relatively high prevalence of this mutation in the Colombian population suggests that other individuals may have undiagnosed subclinical disease.

Key words: Gelatinous drop-like corneal dystrophy, primary familial amyloidosis of the cornea, hereditary corneal dystrophies, corneal amyloidosis. 


\section{Introduction}

Gelatinous drop-like corneal dystrophy (GDCD; MIM 204870) was first described in Japanese patients in $1914 .^{1}$ It is a rare autosomal recessive disease with features consistent with bilateral corneal amyloidosis, leading to pain and severe loss of sight. ${ }^{2,3}$ The majority of reports are from Japan, where the prevalence is estimated to be $1: 300,000$ individuals. ${ }^{4}$ The first case report from outside Japan was from the United States where it was termed primary familial amyloidosis of the cornea. ${ }^{5}$ In Latin America, clinical reports of GDCD cases have been described from Mexico, with 13 cases observed over a 44 year period, and a single case from Brazil. ${ }^{6-8}$

The phenotype of GDCD can gradually develop over time. In the first decade there may only be subepithelial lesions that are similar to band-shaped corneal degeneration, or multiple small grayish epithelial nodules that cause severe photophobia, tearing and foreign body sensation. Over time the nodules increase in number and coalesce to give a mulberry appearance. Finally, in the third decade there is secondary stromal opacification and neovascularization with severe visual loss. ${ }^{9-11}$ The histopathological features include subepithelial and anterior stromal amyloid material deposit with loss of the Bowman layer. The amyloid deposits stain with Congo red, and show birefringence and dichroism under polarized light. ${ }^{12}$ Physiologically, there is increased permeability of the epithelial cell junction. ${ }^{13} \mathrm{Bi}-$ allelic mutations in TACSTD2 (previously M1S1 or TROP2), located on chromosome $1 \mathrm{p} 32$ cause GDCD (MIM 137290). ${ }^{14}$ TACSTD2 encodes tumor associated calcium signal-transducer 2 protein that, in association with claudins 1 and 7 , functions as a transmembrane glycoprotein to maintain the corneal epithelial 
barrier. Loss of this barrier function is associated with accumulation of amyloid in the cornea. ${ }^{15}$

The purpose of this study is to report the clinical features of a consanguineous family with GDCD from Colombia and establish the genetic cause of disease.

\section{MATERIALS AND METHODS}

All participants or their parents provided informed consent for this study. The research was approved by the Ethics Committee of the Medical Research Institute of the University of Antioquia, and adhered to the tenets of the Declaration of Helsinki. A consanguineous family affected with GDCD was examined (Figure 1). A family history and pedigree was constructed and a clinical ophthalmological examination was performed. A therapeutic superficial keratectomy was performed for one patient (individual II:10) and the material was processed for histology. A $10 \mathrm{ml}$ peripheral venous blood sample was taken and DNA extracted (DNeasy, Qiagen () .

The single coding exon of TACSTD2 was amplified by PCR using KapaRobust ${ }^{T M}$ polymerase (Kapa Biosystems) with primers F: 5'-CCTGCAGACCATCCCAGAC-3' and R: 5'-CAGGAAGCGTGACTCACTTG-3'. ${ }^{14}$ PCR products were purified using a MultiScreen ${ }^{\circledR} \mathrm{PCR}_{\mu 96}$ plate (Millipore) and bi-directionally Sanger sequenced. Sequences were aligned and compared to the reference sequence using DNAstar package software version 8.0.2 (Lasergene). Variants were annotated in accordance with Ensembl transcript ID ENST00000371225. 
Two different bioinformatics tools were applied to predict the effect of amino acid substitution on TACSTD2 function: SIFT (v4.0.3; http://sift.jcvi.org/) ${ }^{16}$, which uses sequence homology to make predictions and PolyPhen-2 (v2.0.23; http://genetics.bwh.harvard.edu/pph2/) $)^{17}$, which uses both sequence-based and structure-based predictive features. TACSTD2 variants were checked for their frequency in the Exome Aggregation Consortium database (http://exac.broadinstitute.org/about ) which includes exome data from 63,358 individuals of varying ethnicity.

\section{RESULTS}

The family consisted of consanguineous parents from Caldas in Colombia (Figure 1). The parents had a normal ophthalmic examination. The couple had ten male children, of whom II:1 and II:2 were unavailable for evaluation but had no reported ophthalmic symptoms. Individuals II:6, II:7 and II:11 were clinically unaffected. A maternal uncle was reported to have died blind with opaque corneas of unknown etiology. The son of II:3 (III:1) had no ophthalmic symptoms. Patient II:5 had a welldifferentiated rectal mucinous adenocarcinoma diagnosed at age 22 years, but no other extra-ocular phenotype was noted in any patient.

\section{Clinical findings}

All affected individuals had photophobia, foreign body sensation and blurred vision. There was a significant variation in the phenotype between affected individuals. 
The proband (II: 10) had photophobia and ocular discomfort since the first year of life. Corneal opacities were noted at three years of age, with a visual acuity of count fingers right eye and light perception left eye. By age ten there were dense central corneal opacities with an irregular friable surface with circumferential superficial and deep corneal vascularization (Figure 2A). He had a left superficial keratotomy with an amniotic membrane onlay graft and temporal tarsorrhaphy. After 6 months the visual acuity had improved to 20/200 right eye and 20/400 left eye, with a reduction in opacity (Figure $2 \mathrm{~B}$ ). Histopathology of the excised material showed a stratified squamous corneal epithelium, with preserved polarity and maturation, but with diffuse deposition of an amorphous eosinophilic material in the stroma consistent with amyloid (Supplementary Figure 1). A similar mulberry appearance with neovascularization was noted in individuals II:3 aged 25 years (VA 20/100 BE) and II:5 aged 23 years (VA count fingers OD and hand movements OS) (Figure 2E and 2F). However, in individuals II:8 and II:9 aged 14 and 11 years the visual acuity was preserved (II:8 20/30 OD, 20/25 OS; II:9 20/20 BE). In both these individuals the corneal changes were less severe with only polymorphic grey subepithelial band-shaped opacities without neovascularization, with late epithelial stain following instillation of fluorescein (Figure 2D and 2E). In addition, two affected individuals (II:3 and II:8) also showed peri-limbal deposits suggestive of amyloid material (Figure 2F)

\section{Genetic analysis of TACSTD2}

Screening the TACSTD2 gene identified a single variant; c.551A>G, p.(Y184C) (rs190800473), which fully segregated with the disease in the family. All affected 
individuals were homozygous for the mutation, both unaffected parents (I:1 and $\mathrm{I}: 2$ ), one healthy sibling (II:7) and the son of affected individual II:3 (III:1) were heterozygous for the mutation. All remaining unaffected family members were Homozygous wild-type (Figure 1).

The same mutation has previously been identified in a compound heterozygous state in an affected individual in combination with the Japanese founder mutation c.352C $>\mathrm{T}, \mathrm{p} .(\mathrm{Q} 118 \mathrm{X})$, and is therefore considered to be disease-causing ${ }^{18}$. This is the first report of the mutation in the homozygous state. The TACSTD2 gene is present only in mammals and birds. The non-synonymous variant, p.(Y184C), alters a highly conserved residue (Supplementary Figure 2). Using SIFT and Polyphen2 prediction tools, the p.(Y184C) substitution is predicted to be Damaging (0) and Probably Damaging (0.994), respectively.

The variant was identified only once in the heterozygous state out of 107098 alleles in the Exome Aggregation Consortium (ExAC) database, thus this variant is extremely rare $(\mathrm{MAF}=0.000009337)$. The ExAC data set combines exome sequencing data from large-scale sequencing projects world-wide; the single occurrence of the mutation was detected in an individual of Colombian origin sequenced as part of the 1000 Genomes Project. In total, 93 Colombian individuals were sequenced, therefore the variant has a MAF of $0.53 \%$ in this specific population.

\section{DISCUSSION}


This is the first time, to our knowledge, that GDCD has been genetically solved in patients of Latin American origin (Table 2). The causative homozygous TACSTD2 mutation identified in all affected family members has only previously been identified in the compound heterozygous state in a Chinese patient. ${ }^{18}$

The identification of five affected individuals in this consanguineous family helps to define the phenotypic spectrum associated with the p.(Y184C) TACSTD2 mutation, which shows clinical variability between affected individuals. Two affected individuals (II:8 and II:9), age 14 and 11 years, had a mild phenotype with an appearance similar to band-shaped keratopathy, characteristic of early GDCD. ${ }^{11,13}$ The remaining three affected family members (II:3, II:5 and II:10), age 25, 23 and 10 years, had a more characteristic phenotype with a mulberry appearance and secondary stromal neovascularization of the cornea, associated with severe photophobia, discomfort and blindness. These differences suggest that environmental or other genetic factors contribute to the phenotype, although it is possible that the milder phenotype observed in some individuals in this family could progress over time. Previous reports have indicated that the phenotypic variability for this dystrophy is due to age related progression over time, as the nodular subepithelial amyloid deposits gradually increase in number and coalesce during the first and second decades of life. ${ }^{3,9,10}$ Two affected individuals (II:3 and II:8) also showed peri-limbal deposits suggestive of amyloid material, although confirmation would require a histopathological examination. ${ }^{19}$

GDCD is normally associated with severe ocular morbidity and blindness. Visual rehabilitation is problematic, often requiring multiple surgical interventions, with a 
high rate of recurrence and continued visual decline. After superficial excision of affected material, lamellar or penetrating keratoplasty, temporary improvement can be achieved, but with recurrence at an average of 26 months. ${ }^{20,}{ }^{21}$ Individual II:10 had a superficial keratotomy with an amniotic membrane graft that improved the visual acuity and gave a marked reduction in symptoms for six months, but further follow up is required to confirm the duration of this effect.

Although most reports of GDCD are from East Asia, cases have also been reported from other regions. ${ }^{5}$ To date, 28 different disease-causing mutations in the TACSTD2 gene have been reported, shown in Table 1 with the ethnicity of the affected patients. The majority of mutations are predicted to produce truncated proteins, or proteins degraded by nonsense mediated decay, because of nucleotide substitutions that generate premature stop codons. The remainder are missense changes of conserved residues. The most frequently reported mutation is the c.352C>T, p.(Q118X) mutation which is a founder mutation in the Japanese population, explaining the higher prevalence of GDCD in Japan. ${ }^{14}$

Figure 3 shows a schematic representation of pathogenic TACSTD2 mutations, including the p.(Y184C) mutation, and their location in regards to the functional domains of the TACSTD2 protein. Given the range of previously described missense mutations (see Table 1), it is noteworthy that cysteine residues are often eliminated or created, which might point to a common mechanism of disruption of disulphide bonds in the extracellular domain of the protein. In the cornea, TACSTD2 is localized to epithelial cell junctions and reduction of TACSTD2 
expression has been shown to lead to altered expression and modification of intercellular proteins, including claudins 1,4 , and 7, ZO1 and occludin. ${ }^{15}$ This impairs the function of the epithelial barrier, allowing the formation of amyloid deposits of lactoferrin, apolipoprotein and transforming growth factor $\beta$ (TGFBI). ${ }^{23,24}$ There is evidence that amyloid fibers are formed by non-enzymatic posttranslational modifications of these proteins, including the formation of advanced glycation end products (AGE) and the racemization of aspartyl residues that lead to the misfolding of DNA, resistance to degradation and posterior accumulation. ${ }^{24}$

A single heterozygous carrier of the p.(Y184C) mutation was identified in the 1000 Genomes Project which includes exome sequence data for 93 unrelated individuals from Colombia, suggesting a $0.53 \%$ frequency for the allele in this population. Based on this allele frequency, and excluding inbreeding, we estimated the prevalence of GDCD to be $1: 35,714$ in Colombia, which is 8,5 times the prevalence of GDCD in the Japanese population, for which a founder effect for the p.(Q118X) mutation has been reported. The family is originally from a village in the Department of Caldas in Colombia that has had a rapid expansion in population since its founding. ${ }^{25}$

This is the first clinical, genetic and histopathological description of GDCD from Colombia. The clinical variability of the corneal changes in the affected family members helps define the phenotype of this disease. The most severely affected individual (II:5; 23 years of age) had a history of a colon adenocarcinoma. Although this association has not been highlighted in other reports, a higher expression of 
TACSTD2 has been reported to be associated with aggressive colorectal carcinoma. ${ }^{26}$ It is noteworthy that the relatively high frequency of this allele in ethnically matched control subjects predicts a higher frequency of the disease in Colombia than has been reported in the Japanese population.

\section{Acknowledgements}

The National Institute for Health Research Biomedical Research Centre at Moorfields Eye Hospital NHS Foundation Trust and UCL Institute of Ophthalmology, Fight for Sight, The Lanvern Foundation, Moorfields Eye Charity and Moorfields Special Trustees.

Beatriz Mora MD from the Department of Genetics, Universidad de Antioquia. Medellín, Colombia.

Paula Andrea Marín MD from the Ophthalmology Service, Hospital Universitario San Vicente Fundación. Medellín, Colombia.

\section{Figures}

Figure 1. Pedigree of a Colombian family with GDCD and segregation of a homozygous TACSTD2 missense mutation. The closed symbols represent the individuals diagnosed with GDCD, open symbols are unaffected. The proband (II:10) is marked with an arrow. The letters A and $G$ represent the genotypes for each individual on the complementary strand; $A$ is the wild type allele and $G$ is the mutated allele. ${ }^{*}$ indicates patient has been clinically examined and a DNA sample obtained. No DNA sample was available for patients II:1 and II:2. Individuals II:3, 
$\mathrm{II}: 5$ and II:10 had a severe phenotype (shown in black), while II:8 and II:9 had a milder phenotype with preserved vision (shown in grey).

Figure 2. Differential phenotypic expression of GDCD in affected individuals. (A) Individual II:10 aged 10 years (right eye) with a typical mulberry corneal lesion with superficial vascularization, and (B) (left eye) 6 months after the superficial keratectomy. (C) Individual II:5 aged 23 years (right eye) showing mainly peripheral lesions, some of which are raised with a mulberry appearance accompanied by extensive superficial vascularization. (D) Individual II:8 aged 14 years (left eye) and (E) (right eye) with grey central and paracentral subepithelial band-shaped opacities. (F) Individual II:3 aged 25 years (right eye) with polymorphic whiteyellowish subepithelial corneal and conjunctival lesions with a mulberry appearance and extensive superficial vascularization.

Figure 3. Topology diagram showing location of pathogenic TACSTD2 mutations. Amino acids are numbered at 50 residue intervals. Intracellular, cytosolic and extracellular aspects of the protein are shown, with functional domains and residues (glycosylation sites and disulphide residues) highlighted. All published mutations are mapped to the protein, coloured for functional consequences: missense (yellow), frameshift (grey) and stop (red) mutations.

Supplementary Figure 1. Corneal histology for Individual II:10. (A): Diffuse deposition of amorphous eosinophilic material beneath the epithelium (hematoxylin-eosin). (B) Positive staining for subepithelial amyloid material (Congo 
red).

\section{Supplementary Figure 2. Alignment of p.Tyr184 residue showing} conservation. Alignment of vertebrate TACSTD2 sequences using ClustalW shows conservation of the tyrosine residue shown in bold.

\section{REFERENCES}

1. Nakaizumi G, A rare case of corneal dystrophy. Journal/Nippon Ganka Gakkai Zasshi 1914; 18:945-950.

2. Weiss JS, Moller HU, Lisch W, Kinoshita S, Aldave AJ, Belin MW, Kivela T, Busin M, Munier FL, Seitz B, Sutphin J, Bredrup C, Mannis MJ, Rapuano CJ, Van Rij G, Kim EK and Klintworth GK, The IC3D classification of the corneal dystrophies. Journal/Cornea 2008; 27 Suppl 2(S1-83).

3. Kawasaki S and Kinoshita S, Clinical and basic aspects of gelatinous drop-like corneal dystrophy. Journal/Developments in ophthalmology 2011; 48(97-115.

4. Tsujikawa M, Kurahashi H, Tanaka T, Okada M, Yamamoto S, Maeda N, Watanabe $\mathrm{H}$, Inoue $\mathrm{Y}$, Kiridoshi A, Matsumoto $\mathrm{K}$, Ohashi $\mathrm{Y}$, Kinoshita S, Shimomura $\mathrm{Y}$, Nakamura $\mathrm{Y}$ and Tano $\mathrm{Y}$, Homozygosity mapping of a gene responsible for gelatinous drop-like corneal dystrophy to chromosome $1 \mathrm{p}$. Journal/American journal of human genetics 1998; 63(4):1073-1077.

5. Kirk HQ, Rabb M, Hattenhauer J and Smith R, Primary familial amyloidosis of the cornea. Journal/Transactions - American Academy of Ophthalmology and Otolaryngology. American Academy of Ophthalmology and Otolaryngology 1973; 77(4):OP411-417. 
6. Franco-Díaz de León Alejandro R-RAA, Gómez-Leal Alfredo Amiloidosis familiar congénita. Journal/Revista Mexicana de Oftalmología 2003; 77(2):4.

7. Pineda-Cárdenas Ma. A. Araceli G-AG, Manifestaciones ultraestructurales, histoquímicas e inmunopatológicas de la betafibrilosis corneal primaria. Journal/Revista Mexicana de Oftalmología 2002; 76(4):8.

8. Magalhaes, Otávio de Azevedo et al. Optical coherence tomography image in gelatinous drop-like corneal dystrophy: case report. Journal/Arquivos Brasileiros de Oftalmologia 2012; 75 (5): 356-357

9. Weber FL and Babel J, Gelatinous drop-like dystrophy. A form of primary corneal amyloidosis. Journal/Archives of ophthalmology 1980; 98(1):144-148.

10. Mondino BJ, Rabb MF, Sugar J, Sundar Raj CV and Brown SI, Primary familial amyloidosis of the cornea. Journal/American journal of ophthalmology 1981; 92(5):732-736.

11. Ide T, Nishida K, Maeda N, Tsujikawa M, Yamamoto S, Watanabe H and Tano $\mathrm{Y}, \mathrm{A}$ spectrum of clinical manifestations of gelatinous drop-like corneal dystrophy in japan. Journal/American journal of ophthalmology 2004; 137(6):1081-1084.

12. Klintworth GK, Corneal dystrophies. Journal/Orphanet journal of rare diseases $2009 ; 4(7$.

13. Kinoshita S, Nishida K, Dota A, Inatomi T, Koizumi N, Elliott A, Lewis D, Quantock A and Fullwood N, Epithelial barrier function and ultrastructure of gelatinous drop-like corneal dystrophy. Journal/Cornea 2000; 19(4):551-555.

14. Tsujikawa M, Kurahashi H, Tanaka T, Nishida K, Shimomura $\mathrm{Y}$, Tano $\mathrm{Y}$ and Nakamura $\mathrm{Y}$, Identification of the gene responsible for gelatinous drop-like corneal dystrophy. Journal/Nature genetics 1999; 21(4):420-423. 
15. Nakatsukasa M, Kawasaki S, Yamasaki K, Fukuoka H, Matsuda A, Tsujikawa M, Tanioka H, Nagata-Takaoka M, Hamuro J and Kinoshita S, Tumor-associated calcium signal transducer 2 is required for the proper subcellular localization of claudin 1 and 7: implications in the pathogenesis of gelatinous drop-like corneal dystrophy. Journal/The American journal of pathology 2010; 177(3):1344-1355.

16. $\mathrm{Ng} \mathrm{PC}$ and Henikoff $\mathrm{S}$, Predicting deleterious amino acid substitutions. Journal/Genome Res 2001; 11(5):863-874.

17. Adzhubei IA, Schmidt S, Peshkin L, Ramensky VE, Gerasimova A, Bork P, Kondrashov AS and Sunyaev SR, A method and server for predicting damaging missense mutations. Journal/Nat Methods 2010; 7(4):248-249.

18. Tian X, Fujiki K, Li Q, Murakami A, Xie P, Kanai A, Wang W and Liu Z, Compound heterozygous mutations of M1S1 gene in gelatinous droplike corneal dystrophy. Journal/American journal of ophthalmology 2004; 137(3):567-569.

19. Sayar RB, von Domarus $D$ and Steinhorst $U$, [Spheroid degeneration of the cornea]. Journal/Ophthalmologica. Journal international d'ophtalmologie. International journal of ophthalmology. Zeitschrift fur Augenheilkunde 1986; 193(3):129-134.

20. Shimazaki J, Hida T, Inoue M, Saito H, Tsubota K. Long-term follow-up of patients with familial subepithelial amyloidosis of the cornea. Journal/Ophthalmology 1995 Jan; 102 (1):139-144

21. Uhlig CE, Groppe M, Busse $H$ and Saeger $W$, Morphological and histopathological changes in gelatinous drop-like corneal dystrophy during a 15year follow-up. Journal/Acta ophthalmologica 2010; 88(7):e273-274. 
22. Klintworth GK, Valnickova Z, Kielar RA, Baratz KH, Campbell RJ and Enghild JJ, Familial subepithelial corneal amyloidosis--a lactoferrin-related amyloidosis. Journal/Investigative ophthalmology \& visual science 1997; 38(13):2756-2763.

23. Nishida K, Quantock AJ, Dota A, Choi-Miura NH and Kinoshita S, Apolipoproteins $\mathrm{J}$ and $\mathrm{E}$ co-localise with amyloid in gelatinous drop-like and lattice type I corneal dystrophies. Journal/The British journal of ophthalmology 1999; 83(10):1178-1182.

24. Kaji Y, Oshika T, Takazawa $\mathrm{Y}$, Fukayama M and Fujii N, Co-localisation of advanced glycation end products and D-beta-aspartic acid-containing proteins in gelatinous drop-like corneal dystrophy. Journal/The British journal of ophthalmology 2012; 96(8):1127-1131.

25. Carvajal-Carmona LG, Ophoff R, Service S, Hartiala J, Molina J, Leon P, Ospina J, Bedoya G, Freimer N and Ruiz-Linares A, Genetic demography of Antioquia (Colombia) and the Central Valley of Costa Rica. Journal/Human genetics 2003; 112(5-6):534-541.

26. Ohmachi T, Tanaka F, Mimori K, Inoue H, Yanaga $\mathrm{K}$ and Mori M, Clinical significance of TROP2 expression in colorectal cancer. Journal/Clinical cancer research : an official journal of the American Association for Cancer Research 2006; 12(10):3057-3063. 
Figure 1

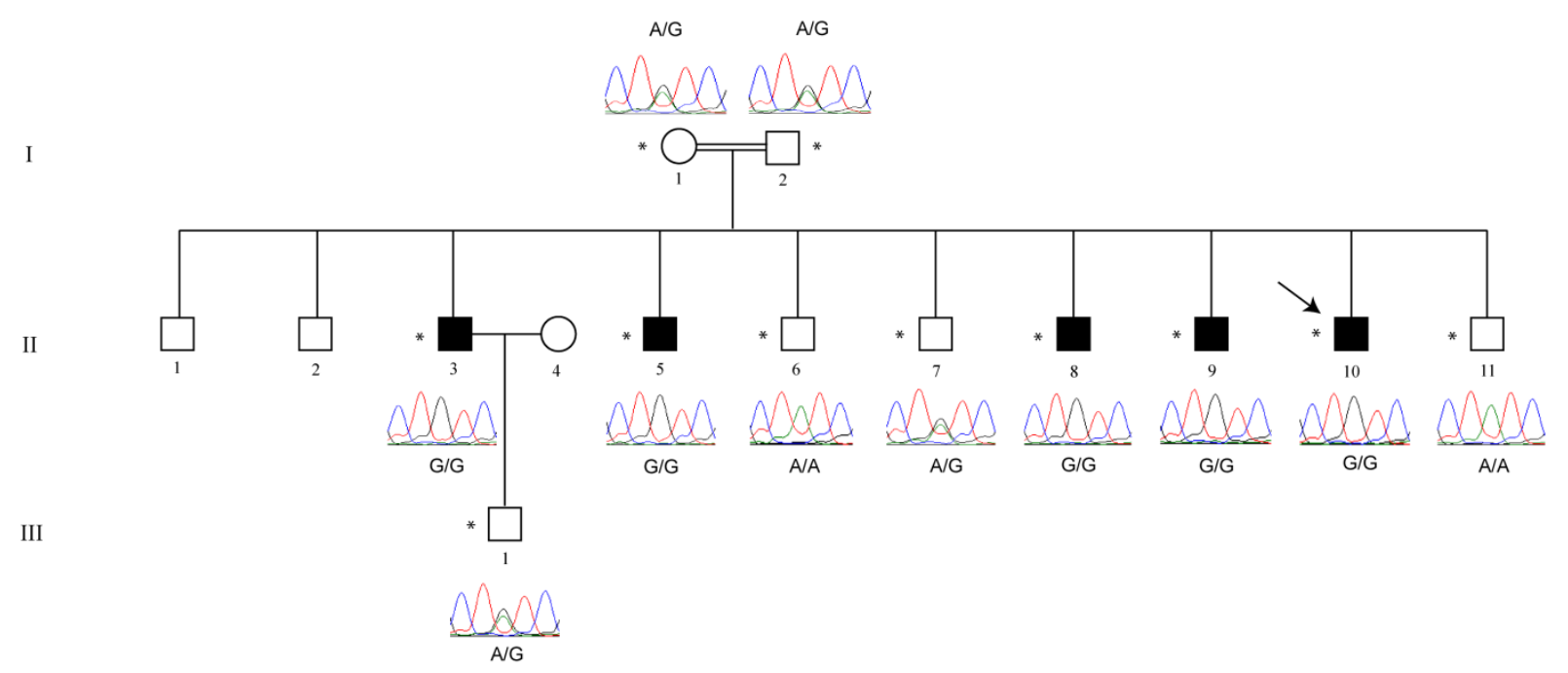

Figure 2

A
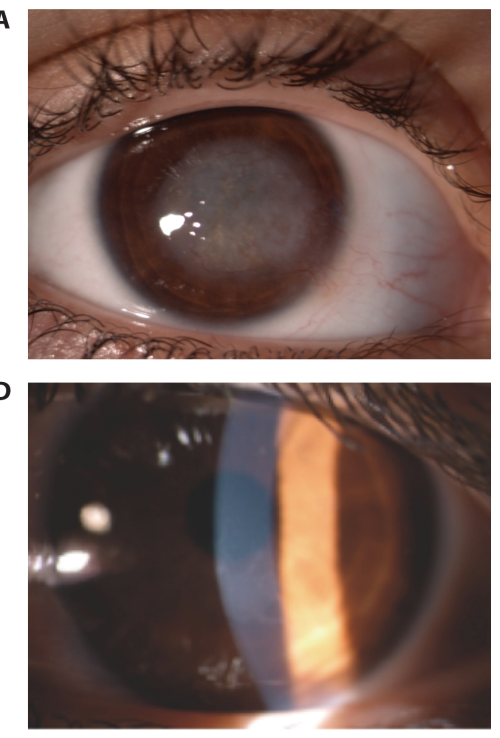
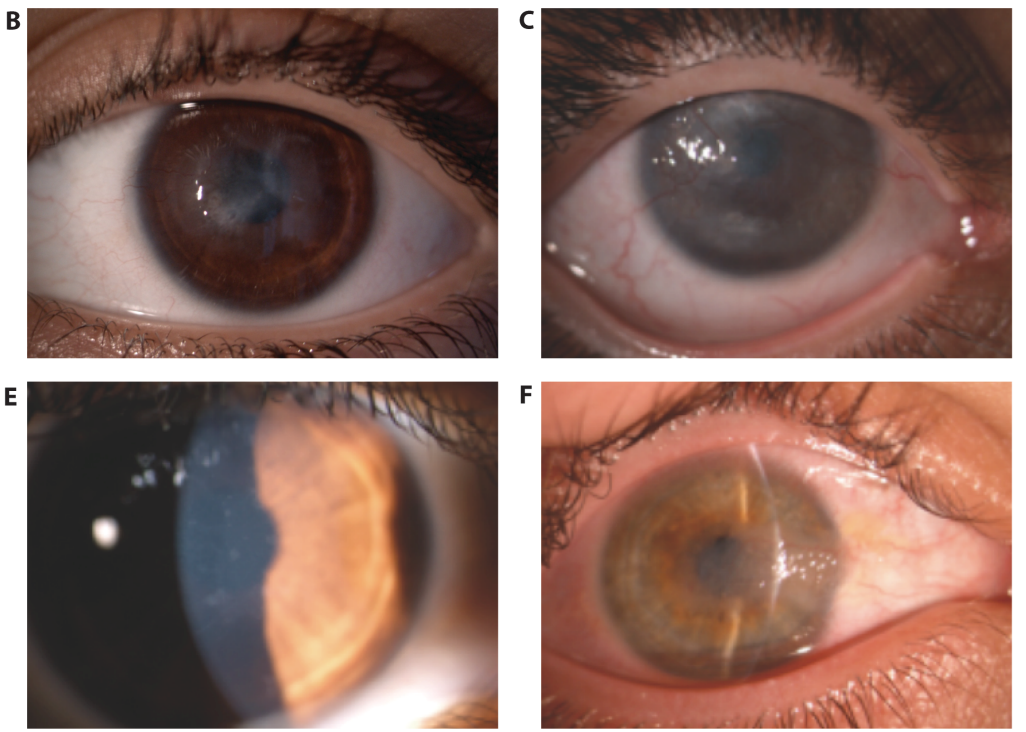
Figure 3

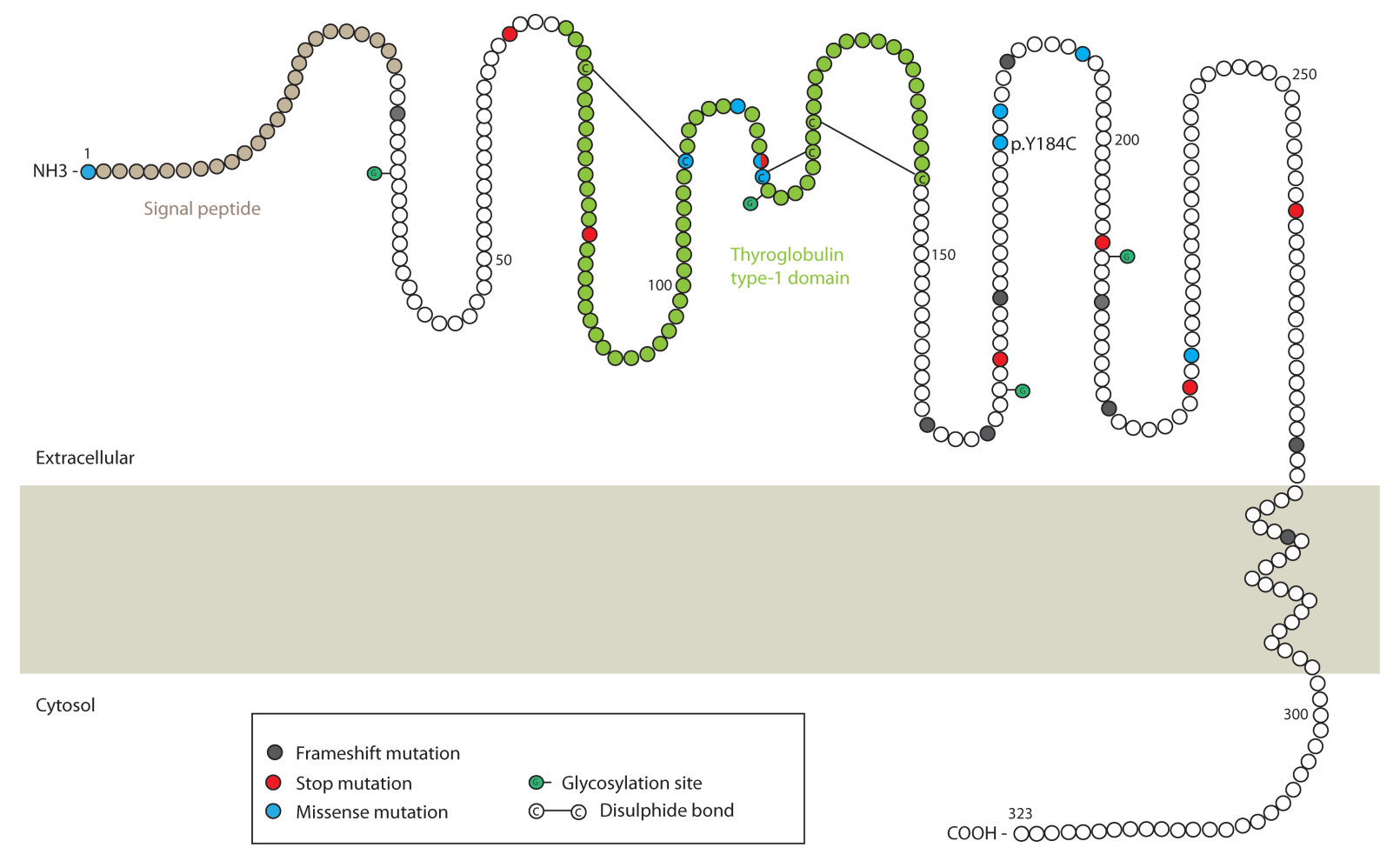




\title{
Supplementary Material
}

\section{Supplementary Figure 1}
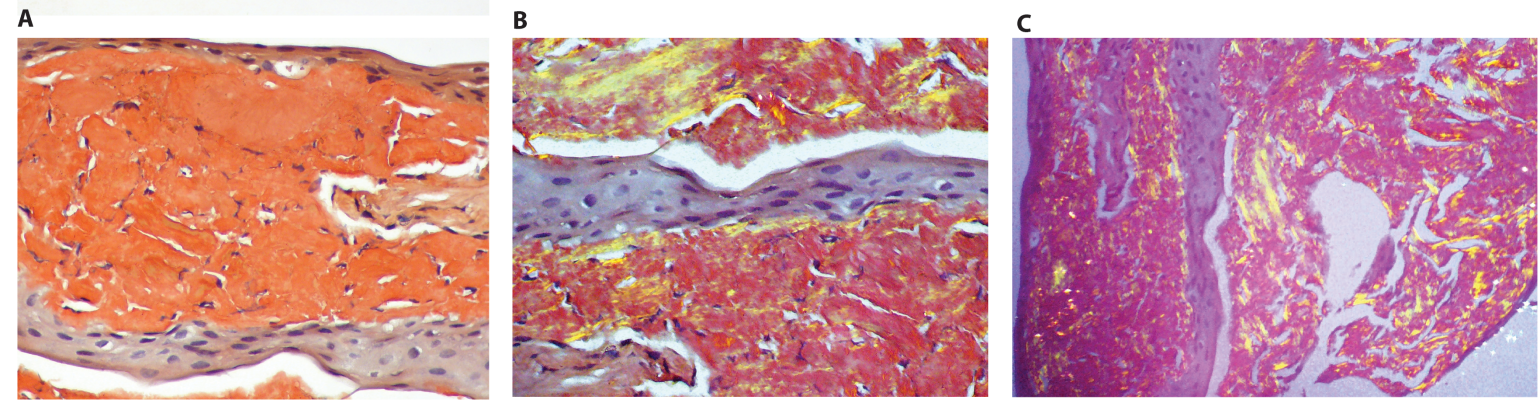

\section{Supplementary Figure 2}

Homo sapiens Pan troglodytes

Macaca mulatta Rattus norvegic Gallus gallus

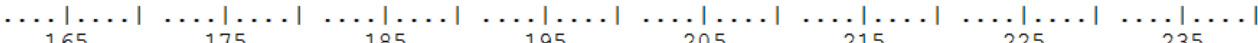
HRPTAGAFNH SDLDAELRRL FRERYRLHPK FVAAVHYEQP TIQIELRQNT SQKAAGDVDI GDAAYYFERD IKGESLFQGR HRPTAGAFNH SDLDAELRRL FRERYRLHPK FVAAVHYEQP TIQIELRQNT SQKAAGDVDI GDAAYYFERD IKGESLFQGR HRPTAGAFNH SDLDAELRRL FRERYRLHPK FVAAVHYEOP TIOIELRONT SOKAAGDVDI GDAAYYFERD VKGESLFQGR HRPTDRAFNH SDLDSELRRL FKERYKLHPS FLAAVHYEEP TIQIELQQNA SQKGLRDVDI ADAAYYFERD IKGESLFVGR HKETSHYFEA LDVAEALTHL FENRYKLQPK YIAAVKYDSP FIQIHLKQNK Q----CDIDI VDVAYYLEKD VKGDSVFHSD
\end{abstract}

